\title{
FINITE $p$-GROUPS WHICH ARE NOT GENERATED BY THEIR NON-NORMAL SUBGROUPS
}

\author{
ZVONIMIR JANKO \\ University of Heidelberg, Germany
}

\begin{abstract}
Here we classify finite non-Dedekindian $p$-groups which are not generated by their non-normal subgroups. (Theorem 1).
\end{abstract}

The purpose of this paper is to classify non-Dedekindian finite $p$-groups which are not generated by their non-normal subgroups. It is surprising that such $p$-groups must be of class 2 with a cyclic commutator subgroup.

We consider here only finite $p$-groups and our notation is standard (see [1]). We prove the following result.

TheOREM 1. Let $G$ be a non-Dedekindian p-group and let $G_{0}$ be the subgroup generated by all nonnormal subgroups of $G$, where we assume $G_{0}<G$. Then $G$ is of class $2, G / G_{0}$ is cyclic and for each $g \in G-G_{0}$, $\{1\} \neq\langle g\rangle \cap G_{0} \unlhd G$ and $G /\left(\langle g\rangle \cap G_{0}\right)$ is abelian so that $G^{\prime}$ is cyclic.

Proof. Since our group $G$ has at least $p$ (non-normal) conjugate cyclic subgroups, it follows that the subgroup $G_{0}$ is noncyclic. Let $x \in G-G_{0}$. Then $\langle x\rangle \unlhd G$, by hypothesis, and so $G^{\prime}$ centralizes $\langle x\rangle$. It follows from $\left\langle G-G_{0}\right\rangle=G$ that $G^{\prime} \leq \mathrm{Z}(G)$ and $\operatorname{so} \operatorname{cl}(G)=2$.

Let $g \in G-G_{0}$. Then $Z=\langle g\rangle \triangleleft G$. Write $Z_{0}=Z \cap G_{0}$; then $Z_{0}$, being the intersection of two $G$-invariant subgroups, is $G$-invariant. We claim that $G / Z_{0}$ is Dedekindian. Indeed, let $X / Z_{0}$ be any proper subgroup in $G / Z_{0}$. We have to show that $X \triangleleft G$. If $X \not \leq G_{0}$, then $X \unlhd G$. Now assume that $X<G_{0}$ (the subgroup $G_{0}$ is $G$-invariant). Then $X Z=Z X$ is normal in $G$

2010 Mathematics Subject Classification. $20 \mathrm{D} 15$.

Key words and phrases. Finite p-groups, Dedekindian p-groups, non-normal subgroups. 
since $X Z \not \leq G_{0}$. By the product formula, one has

$$
\left|X Z G_{0}\right|=\left|Z G_{0}\right|=\frac{|Z|\left|G_{0}\right|}{\left|Z_{0}\right|} .
$$

On the other hand,

$$
\left|X Z G_{0}\right|=\frac{|X Z|\left|G_{0}\right|}{\left|X Z \cap G_{0}\right|}=\frac{|X||Z|}{\left|Z_{0}\right|} \cdot \frac{\left|G_{0}\right|}{\left|X Z \cap Z_{0}\right|}=\left|X Z G_{0}\right| \cdot \frac{|X|}{\left|X Z \cap G_{0}\right|}
$$

which implies $X=X Z \cap G_{0} \triangleleft G$, and we are done. We have proved that $G / Z_{0}$ is Dedekindian. In particular, $Z_{0} \neq\{1\}$ since $G$ is non-Dedekindian, by hypothesis. If $p>2$, then $G / Z_{0}$ is abelian and so $G^{\prime} \leq Z_{0}$ and $G^{\prime}$ is cyclic. If $p=2$, then $G / Z_{0}$ is either abelian or Hamiltonian (= nonabelian Dedekindian).

It follows from the above that $\Omega_{1}(G) \leq G_{0}$.

Now assume that $p>2$. Amongst all elements in the set $G-G_{0}$, we choose an element $a$ of the smallest possible order. Then $a^{p} \in G_{0}$ and $G^{\prime} \leq\left\langle a^{p}\right\rangle$ (see the previous paragraph). We set $\left|G^{\prime}\right|=p^{d}, d \geq 1$. Suppose that $G / G_{0}$ is not cyclic. Then there is $b \in G-\left(G_{0}\langle a\rangle\right)$ such that $b^{p} \in G_{0}$. We have $\langle a\rangle \cap\langle b\rangle \geq G^{\prime}$ and $\mathrm{o}(b) \geq \mathrm{o}(a)$ by the minimality of $\mathrm{o}(a)$. Set

$$
|\langle a\rangle /(\langle a\rangle \cap\langle b\rangle)|=p^{s}, \text { where } s \geq 1 \text { and } \mathrm{o}(a) \geq p^{d+s} \text {. }
$$

Hence there is $b^{\prime} \in\langle b\rangle-\langle a\rangle$ such that $a^{p^{s}}=\left(b^{\prime}\right)^{-p^{s}}$. In that case, since $\operatorname{cl}(G)=2$, one obtains

$$
\left(a b^{\prime}\right)^{p^{s}}=a^{p^{s}}\left(b^{\prime}\right)^{p^{s}}\left[b^{\prime}, a\right]^{\left(\begin{array}{c}
p^{s} \\
2
\end{array}\right)}=\left[b^{\prime}, a\right]^{\left(\begin{array}{c}
p^{s} \\
2
\end{array}\right)},
$$

where $s \geq 1, \mathrm{o}(a) \geq p^{d+s}$ and $\left\langle\left[b^{\prime}, a\right]^{\left(\begin{array}{c}p^{s} \\ 2\end{array}\right)}\right\rangle<G^{\prime}$ so that $\mathrm{o}\left(\left[b^{\prime}, a\right]^{\left(\begin{array}{c}p^{s} \\ 2\end{array}\right)}\right)<p^{d}$. It follows that

$$
\mathrm{o}\left(a b^{\prime}\right)<p^{d+s} \text { and so o }\left(a b^{\prime}\right)<\mathrm{o}(a) .
$$

If $b^{\prime} \in\left\langle b^{p}\right\rangle \leq G_{0}$, then $a b^{\prime} \in G-G_{0}$. If $\left\langle b^{\prime}\right\rangle=\langle b\rangle$, then $a b^{\prime} \in G-\left(G_{0}\langle a\rangle\right)$ and so again $a b^{\prime} \in G-G_{0}$. But this contradicts the minimality of o( $\left.a\right)$. We have proved that in case $p>2, G / G_{0}$ is cyclic.

Suppose $p=2$ and $G / G_{0}$ is nonabelian. Then for each $g \in G-G_{0}$, $G /\left(\langle g\rangle \cap G_{0}\right)$ is Hamiltonian (i.e., Dedekindian nonabelian). Let $Q / G_{0}$ be a subgroup of $G / G_{0}$ which is isomorphic to $Q_{8}$ and let $R / G_{0}$ be a unique subgroup of order 2 in $Q / G_{0}$. Then for each $x \in Q-R, x^{2} \in R-G_{0}$. Let $a, b \in Q-R$ be such that $\langle a, b\rangle$ covers $Q / R \cong \mathrm{E}_{4}$. Note that $\langle a\rangle \unlhd G,\langle b\rangle \unlhd G$ and since $\langle a\rangle \cap G_{0} \neq\{1\}$ and $\langle b\rangle \cap G_{0} \neq\{1\}$, we get o $(a)=2^{s}, s \geq 3$, and $\mathrm{o}(b) \geq 2^{3}$. Because

$$
[a, b] \in R-G_{0} \text { and }[a, b] \in\langle a\rangle \cap\langle b\rangle,
$$

we have

$$
\langle a\rangle \cap\langle b\rangle=\left\langle a^{2}\right\rangle=\left\langle b^{2}\right\rangle=\langle[a, b]\rangle .
$$


But then $C=\langle a, b\rangle$ is a 2-group of maximal class and order $2^{s+1}, s \geq 3$, and in this case $\langle a\rangle$ is a unique cyclic subgroup of order $2^{s}$ in $C$, contrary to the fact that $\mathrm{o}(b)=2^{s}$. We have proved that in case $p=2, G / G_{0}$ must be abelian and so $G^{\prime} \leq G_{0}$.

Suppose that $G^{\prime}$ is noncyclic. By the above, $p=2$ and for each $g \in$ $G-G_{0},\{1\} \neq\langle g\rangle \cap G_{0} \unlhd G$, where $G /\left(\langle g\rangle \cap G_{0}\right)$ is Hamiltonian (=nonabelian Dedekindian). Set $D=\langle g\rangle \cap G_{0}$ and $R / D=(G / D)^{\prime} \cong \mathrm{C}_{2}$, where $R=G^{\prime} D$. We know that $G^{\prime} \leq G_{0}$ (since $G / G_{0}$ is abelian) and so $R \leq G_{0}$ and $G / R$ is elementary abelian. In particular, $G / G_{0} \neq\{1\}$ is elementary abelian and $\left\langle g^{2}\right\rangle=D$. Note that all quaternion subgroups in a Hamiltonian 2-group $X$ generate $X$. Hence there is a quaternion subgroup $K / D \cong \mathrm{Q}_{8}$ in the Hamiltonian group $G / D$ such that $K \not \leq G_{0}$. We have $K>R$ and $K / R \cong \mathrm{E}_{4}$ so that for each $x \in K-R, x^{2} \in R-D$. We may choose some elements $a, b \in K-G_{0}$ such that $Q=\langle a, b\rangle$ covers $K / R$ and so $Q$ also covers $K / D$. Note that $\langle a\rangle \unlhd G,\langle b\rangle \unlhd G$ and $[a, b] \in R-D$. Also,

$$
[a, b] \in\langle a\rangle \cap\langle b\rangle \text { and so }\langle[a, b]\rangle=\left\langle a^{2}\right\rangle=\left\langle b^{2}\right\rangle=\langle a\rangle \cap\langle b\rangle \text {. }
$$

This gives $\left|Q: Q^{\prime}\right|=4$ and so (by a well known result of O. Taussky) $Q$ is a 2group of maximal class with two distinct cyclic subgroups $\langle a\rangle$ and $\langle b\rangle$ of index 2. By inspection of 2-groups of maximal class (and noting that $G$ is of class 2), we get $\mathrm{o}(a)=\mathrm{o}(b)=4$ and $Q \cong \mathrm{Q}_{8}$ with $Q^{\prime}=\left\langle a^{2}\right\rangle=\left\langle b^{2}\right\rangle$. Hence $K=Q \times D$ since $Q \unlhd G$ and $Q$ covers $K / D \cong \mathrm{Q}_{8}$. Also, $\langle g\rangle \unlhd G$ and $Q \cap\langle g\rangle=\{1\}$ and so $Q$ centralizes $\langle g\rangle$. The factor-group $G /\left\langle a^{2}\right\rangle$ is Hamiltonian and so

$$
\mathrm{o}(g)=4, D=\left\langle g^{2}\right\rangle \cong \mathrm{C}_{2} \text { and } G^{\prime}=\left\langle a^{2}, g^{2}\right\rangle \cong \mathrm{E}_{4}
$$

since $G^{\prime}$ covers $\left\langle a^{2}, g^{2}\right\rangle /\left\langle a^{2}\right\rangle$ and $G^{\prime}$ is noncyclic. For each $x \in G$,

$$
x^{4} \in\left\langle a^{2}\right\rangle \cap\left\langle g^{2}\right\rangle=\{1\} \text { and so } \exp (G)=4 .
$$

Let $K_{1} /\left\langle a^{2}\right\rangle \cong \mathrm{Q}_{8}$ with $K_{1} \not G_{0}$. Then choose $a_{1}, b_{1} \in K_{1}-G_{0}$ such that $\left\langle a_{1}, b_{1}\right\rangle$ covers $K_{1} /\left\langle a^{2}\right\rangle$. We get

$$
\begin{gathered}
Q_{1}=\left\langle a_{1}, b_{1}\right\rangle \cong \mathrm{Q}_{8} \text { with } Q \cap Q_{1}=\{1\} \text { and } Q_{1}^{\prime}=\left\langle a_{1}^{2}\right\rangle=\left\langle b_{1}^{2}\right\rangle, \\
\text { so }\left\langle Q, Q_{1}\right\rangle=Q \times Q_{1} .
\end{gathered}
$$

Set $a^{2}=t, a_{1}^{2}=t_{1}$ and let $x \in Q-\langle t\rangle, x_{1} \in Q_{1}-\left\langle t_{1}\right\rangle$ so that $x x_{1}$ is one of 36 elements of order 4 with $\left(x x_{1}\right)^{2}=x^{2} x_{1}^{2}=t t_{1}$. We claim that $\left\langle x x_{1}\right\rangle$ is not normal in $Q \times Q_{1}$ and so $x x_{1} \in G_{0}$. Indeed, let $y \in Q-\langle x\rangle$ so that

$$
\left(x x_{1}\right)^{y}=x^{-1} x_{1}=\left(x x_{1}\right) t, \text { where }\left(x x_{1}\right) t \notin\left\langle x x_{1}\right\rangle .
$$

But all these 36 elements of order 4 generate $Q \times Q_{1}$ (of order 64) and so $Q \times Q_{1} \leq G_{0}$, a contradiction. We have proved that also in case $p=2, G^{\prime}$ is cyclic.

In the following five paragraphs we assume that $G / G_{0}$ is noncyclic. By the above, $p=2$ and $G / G_{0}$ is abelian. 
Assume that there are $a_{1}, a_{2} \in G-G_{0}$ such that $\left\langle a_{1}\right\rangle \cap\left\langle a_{2}\right\rangle=\{1\}$. We know that $G /\left(\left\langle a_{1}\right\rangle \cap G_{0}\right)$ and $G /\left(\left\langle a_{2}\right\rangle \cap G_{0}\right)$ are Dedekindian and $\left[a_{1}, a_{2}\right] \in$ $\left\langle a_{1}\right\rangle \cap\left\langle a_{2}\right\rangle=\{1\}$ and so $\left\langle a_{1}, a_{2}\right\rangle$ is abelian. If both $G /\left(\left\langle a_{1}\right\rangle \cap G_{0}\right)$ and $G /\left(\left\langle a_{2}\right\rangle \cap G_{0}\right)$ are abelian, then

$$
G^{\prime} \leq\left(\left\langle a_{1}\right\rangle \cap G_{0}\right) \cap\left(\left\langle a_{2}\right\rangle \cap G_{0}\right)=\{1\},
$$

a contradiction. Assume for a moment that both $G /\left(\left\langle a_{1}\right\rangle \cap G_{0}\right)$ and $G /\left(\left\langle a_{2}\right\rangle \cap\right.$ $\left.G_{0}\right)$ are Hamiltonian. Then for each $x \in G$,

$$
x^{4} \in\left(\left\langle a_{1}\right\rangle \cap G_{0}\right) \cap\left(\left\langle a_{2}\right\rangle \cap G_{0}\right)=\{1\} \text { and so } \exp (G)=4 .
$$

In particular,

$$
\mathrm{o}\left(a_{1}\right)=\mathrm{o}\left(a_{2}\right)=4,\left\langle a_{1}^{2}, a_{2}^{2}\right\rangle \cong \mathrm{E}_{4} \text { with }\left\langle a_{1}^{2}, a_{2}^{2}\right\rangle \leq \mathrm{Z}(G) .
$$

We have

$$
G^{\prime} \leq\left\langle a_{1}^{2}, a_{2}^{2}\right\rangle, G^{\prime} \text { covers }\left\langle a_{1}^{2}, a_{2}^{2}\right\rangle /\left\langle a_{1}^{2}\right\rangle \text { and }\left\langle a_{1}^{2}, a_{2}^{2}\right\rangle /\left\langle a_{2}^{2}\right\rangle \text { and } G^{\prime} \text { is cyclic }
$$

and so $G^{\prime}=\left\langle a_{1}^{2} a_{2}^{2}\right\rangle$. For each

$$
x \in G, \quad\left[a_{2}, x\right] \in\left\langle a_{2}\right\rangle \cap G^{\prime}=\{1\} \text { and so } a_{2} \leq \mathrm{Z}(G) .
$$

But then in the Hamiltonian 2-group $G /\left\langle a_{1}^{2}\right\rangle$ the element $\left(\left\langle a_{2}\right\rangle\left\langle a_{1}^{2}\right\rangle\right) /\left\langle a_{1}^{2}\right\rangle \cong$ $\mathrm{C}_{4}$ of order 4 lies in its center, a contradiction. We have proved that if $a_{1}, a_{2} \in G-G_{0}$ are such that $\left\langle a_{1}\right\rangle \cap\left\langle a_{2}\right\rangle=\{1\}$, then one of $G /\left(\left\langle a_{1}\right\rangle \cap G_{0}\right)$ and $G /\left(\left\langle a_{2}\right\rangle \cap G_{0}\right)$ is abelian and the other one is Hamiltonian.

Assume in addition that $\left(G_{0}\left\langle a_{1}, a_{2}\right\rangle\right) / G_{0}$ is noncyclic. Set $\Omega_{1}\left(\left\langle a_{1}\right\rangle\right)=\left\langle t_{1}\right\rangle$ and $\Omega_{1}\left(\left\langle a_{2}\right\rangle\right)=\left\langle t_{2}\right\rangle$ so that $\left\langle t_{1}, t_{2}\right\rangle \cong \mathrm{E}_{4}$ and $\left\langle t_{1}, t_{2}\right\rangle \leq \mathrm{Z}(G)$. Without loss of generality we may suppose that $G /\left(\left\langle a_{1}\right\rangle \cap G_{0}\right)$ is abelian and $G /\left(\left\langle a_{2}\right\rangle \cap G_{0}\right)$ is Hamiltonian. Since $G / G_{0}$ is elementary abelian, we get

$$
\mathrm{o}\left(a_{1}\right)=4, G^{\prime}=\left\langle a_{1}^{2}\right\rangle \cong \mathrm{C}_{2} \text { and } 1 \neq a_{2}^{2} \in G_{0} .
$$

It follows that $\left(G_{0}\left\langle a_{1}, a_{2}\right\rangle\right) / G_{0} \cong \mathrm{E}_{4}$. Let $a_{2}^{\prime}$ be an element of order 4 in $\left\langle a_{2}\right\rangle$ so that

$$
\left(a_{1} a_{2}^{\prime}\right)^{2}=a_{1}^{2}\left(a_{2}^{\prime}\right)^{2}=t_{1} t_{2} \text { and } a_{1} a_{2}^{\prime} \in G-G_{0} .
$$

But then $\left\langle a_{1}\right\rangle,\left\langle a_{2}\right\rangle,\left\langle a_{1} a_{2}^{\prime}\right\rangle$ are three cyclic subgroups in $G$ which are not contained in $G_{0}$ and they have pairwise a trivial intersection. By the previous paragraph, this is not possible. We have proved that whenever $a_{1}, a_{2} \in G-G_{0}$ are such that $\left(\left\langle a_{1}, a_{2}\right\rangle G_{0}\right) / G_{0}$ is noncyclic, then $\left\langle a_{1}\right\rangle \cap\left\langle a_{2}\right\rangle \neq\{1\}$.

Let $E / G_{0}$ be a four-subgroup in the noncyclic abelian group $G / G_{0}$. Amongst all elements in $E-G_{0}$ choose an element $a$ of the smallest possible order $2^{s}$. We have $s \geq 2$ since $a^{2} \neq 1$. Set $F=G_{0}\langle a\rangle$ and let $b$ be any element in $E-F$ so that o $(b) \geq 2^{s}$. By the above, $D=\langle a\rangle \cap\langle b\rangle \neq\{1\}$. Let $b^{\prime}$ be an element of order $2^{s}$ in $\langle b\rangle$ such that

$$
\begin{gathered}
a^{2^{n}}=\left(b^{\prime}\right)^{-2^{n}}, \text { where }|\langle a\rangle: D|=\left|\left\langle b^{\prime}\right\rangle: D\right|=2^{n}, n \geq 1, \\
\text { and } D=\left\langle a^{2^{n}}\right\rangle=\left\langle\left(b^{\prime}\right)^{2^{n}}\right\rangle .
\end{gathered}
$$


We compute

$$
\begin{aligned}
& \left(a b^{\prime}\right)^{2^{n}}=a^{2^{n}}\left(b^{\prime}\right)^{2^{n}}\left[b^{\prime}, a\right]^{\left(\begin{array}{c}
2^{n} \\
2
\end{array}\right)}=\left[b^{\prime}, a\right]^{2^{n-1}\left(2^{n}-1\right)}, \\
& \text { where } a b^{\prime} \in E-G_{0} \text { and }\left[b^{\prime}, a\right] \in D \text {. }
\end{aligned}
$$

Since $a$ was an element of the smallest possible order in the set of all elements in $E-G_{0}$, we get

$$
n=1, a^{2} \in D, \text { and }\left\langle\left[b^{\prime}, a\right]\right\rangle=D \neq\{1\} .
$$

On the other hand,

$$
[b, a]^{2}=\left[b, a^{2}\right]=1 \text { and so }\left[b^{2}, a\right]=[b, a]^{2}=1 .
$$

Hence, if $b^{\prime} \in\left\langle b^{2}\right\rangle$ (in case o $\left.(b)>\mathrm{o}(a)=2^{s}\right)$, we get $\left[b^{\prime}, a\right]=1$ and so $D=\{1\}$, a contradiction. It follows that

$$
\mathrm{o}(a)=\mathrm{o}(b)=2^{s} \text { and }\langle[b, a]\rangle=D \cong \mathrm{C}_{2} \text {. where } D=\left\langle a^{2}\right\rangle=\left\langle b^{2}\right\rangle .
$$

Hence

$$
s=2, \mathrm{o}(a)=\mathrm{o}(b)=4 \text {, and } Q=\langle a, b\rangle \cong \mathrm{Q}_{8} .
$$

We have proved that all elements in $E-F$ are of order 4 and each such element has the same square $a^{2}$. We know that $G^{\prime}$ is cyclic, $G^{\prime} \leq G_{0}, G^{\prime} \leq \mathrm{Z}(G)$ and $G^{\prime} \geq\left\langle a^{2}\right\rangle=\langle a, b\rangle^{\prime}$. Suppose that $\left.G^{\prime}\right\rangle\left\langle a^{2}\right\rangle$ and let $x \in G^{\prime}-\left\langle a^{2}\right\rangle$ be such that $x^{2}=a^{2}$, where $[x, a]=1$. But then $x a$ is an involution in $E-G_{0}$, a contradiction. Hence $G^{\prime}=\left\langle a^{2}\right\rangle \cong \mathrm{C}_{2}$. Since all elements in $E-F$ are of order 4 and they generate $E$ and $E^{\prime}=\left\langle a^{2}\right\rangle \cong \mathrm{C}_{2}$, we get $\exp (G)=4$. In particular, all elements in $F-G_{0}$ are of order 4 and let $y \in F-G_{0}$. Then $y$ is also of the smallest possible order 4 in $E-G_{0}$. By repeating the above argument with the element $y$ (instead of $a$ ), we get that for each $b \in E-F$, $b^{2}=y^{2}$ and so $y^{2}=a^{2}$. We have proved that for each $x \in E-G_{0}, x^{2}=a^{2}$. For any $x, y \in G$,

$$
\left[x^{2}, y\right]=[x, y]^{2}=1 \text { since } G^{\prime}=\left\langle a^{2}\right\rangle \cong \mathrm{C}_{2} .
$$

Hence $\mho_{1}(G) \leq \mathrm{Z}(G)$

Let $c$ be an element of order 4 in $G_{0}$. Then

$$
\begin{gathered}
a c \in E-G_{0} \text { and so } a^{2}=(a c)^{2}=a^{2} c^{2}[c, a] \\
\text { implying } c^{2}=[a, c] \in\left\langle a^{2}\right\rangle \text { and } c^{2}=a^{2} .
\end{gathered}
$$

But then $\langle c\rangle \unlhd G$ and so there is $b \in E-G_{0}$ which centralizes $\langle c\rangle$. It follows that $b c$ is an involution in $E-G_{0}$, a contradiction. We have proved that $G_{0}$ is elementary abelian. If $G_{0} \not \leq \mathrm{Z}(E)$, then there are $t \in G_{0}-\left\langle a^{2}\right\rangle$ and $x \in E-G_{0}$ such that $[t, x]=a^{2}=x^{2}$. But then $\langle t, x\rangle \cong \mathrm{D}_{8}$ and so there are involutions in $\langle t, x\rangle-G_{0}$, a contradiction. We have proved that $E$ is Hamiltonian and so $E \neq G$ because $G$ is not Dedekindian.

Let $v \in G-E$ be such that $v^{2} \in E$. Since $\mho_{1}(G) \leq \mathrm{Z}(G)$, we get $1 \neq v^{2} \in \mathrm{Z}(E)=G_{0}$. Then, by the above, $\langle v\rangle \cap\langle a\rangle \neq\{1\}$ and so $v^{2}=a^{2}$. 
Let $a, b \in E-G_{0}$ be such that $\langle a, b\rangle$ covers $E / G_{0}$. Because there are no involutions in $G-G_{0}$, we have

$$
[v, a]=[v, b]=[a, b]=a^{2} \text { and }[v, a b]=[v, a]=[v, b]=a^{2} a^{2}=a^{4}=1 .
$$

But then $(a b)^{2}=v^{2}=a^{2}$ implies that $(a b) v$ is an involution in $G-G_{0}$, a final contradiction. We have proved that also in case $p=2, G / G_{0}$ is cyclic.

Suppose that $p=2$ and there is $g \in G-G_{0}$ such that $G /\left(\langle g\rangle \cap G_{0}\right)$ is Hamiltonian. We set $D=\langle g\rangle \cap G_{0} \neq\{1\}$ and note that $G^{\prime} \leq G_{0}$ implies that $G / G_{0}$ is elementary abelian. But $G / G_{0}$ is also cyclic and so $\left|G: G_{0}\right|=2$. We get $g^{2} \in G_{0}$ and so $D=\left\langle g^{2}\right\rangle \neq\{1\}$. Since the Hamiltonian group $G / D$ is generated by its quaternion subgroups, there is a quaternion subgroup $K / D$ in $G / D$ such that $K \not \leq G_{0}$. Let $a, b \in K-G_{0}$ be such that $Q=\langle a, b\rangle$ covers $K / D$, where $a b \in G_{0}$. Let $R / D$ be a unique subgroup of order 2 in $K / D$ so that $R \leq G_{0}$ and $G^{\prime}$ covers $R / D$. We have

$$
a^{2} \in R-D, b^{2} \in R-D,(a b)^{2} \in R-D, \text { and }[a, b] \in R-D .
$$

On the other hand,

$$
[a, b] \in\langle a\rangle \cap\langle b\rangle \text { and so }\langle a\rangle \cap\langle b\rangle=\left\langle a^{2}\right\rangle=\left\langle b^{2}\right\rangle=\langle[a, b]\rangle \text {. }
$$

Since $Q / Q^{\prime} \cong \mathrm{E}_{4}, Q$ is of maximal class (by O. Taussky) and since $Q$ has two distinct cyclic subgroups $\langle a\rangle$ and $\langle b\rangle$ of index 2 , we get

$$
\begin{gathered}
Q \cong \mathrm{Q}_{8}, \mathrm{o}(a)=\mathrm{o}(b)=4,\langle[a, b]\rangle \cong \mathrm{C}_{2}, Q \cap\left\langle g^{2}\right\rangle=\{1\} \\
\text { and so }\langle Q,\langle g\rangle\rangle=Q \times\langle g\rangle .
\end{gathered}
$$

Also,

$$
G^{\prime} \leq R \text { and } G^{\prime} \geq\langle[a, b]\rangle \cong \mathrm{C}_{2},
$$

and so the fact that $G^{\prime}$ is cyclic implies $G^{\prime} \cap\left\langle g^{2}\right\rangle=\{1\}$. It follows

$$
G^{\prime}=\langle[a, b]\rangle=\left\langle a^{2}\right\rangle \cong \mathrm{C}_{2}
$$

and for any $x, y \in G$,

$$
\left.\left[x^{2}, y\right]=[x, y]^{2}=1 \text { implying } \mho_{1} G\right) \leq \mathrm{Z}(G) .
$$

Since $G^{\prime} \cap\langle g\rangle=\{1\}$, we have $\langle g\rangle \leq \mathrm{Z}(G)$ and so $G=G_{0} *\langle g\rangle$ gives that $G_{0}$ is nonabelian. We have $a b \in G_{0}$ and so $a b g \notin G_{0}$ which implies $\langle a b g\rangle \unlhd G$. We compute

If $g^{4} \neq 1$, then

$$
(a b g)^{2}=(a b)^{2} g^{2}=a^{2} g^{2} \text {. }
$$

$$
(a b g)^{4}=g^{4} \neq 1 \text { and so } G^{\prime}=\left\langle a^{2}\right\rangle \not \leq\langle a b g\rangle \text {. }
$$

If $g^{4}=1$, then $a^{2} g^{2}$ is an involution distinct from $a^{2}$ and so again $G^{\prime} \leq\langle a b g\rangle$. It follows that in any case $G^{\prime} \not \leq\langle a b g\rangle$ and so $\langle a b g\rangle \leq \mathrm{Z}(G)$. But then

$$
a b \in \mathrm{Z}(G) \text { giving } \mathrm{C}_{4} \cong\langle a b\rangle \leq \mathrm{Z}(Q),
$$

a contradiction. We have proved that for each $g \in G-G_{0}, G /\left(\langle g\rangle \cap G_{0}\right)$ is abelian. Our theorem is proved. 


\section{REFERENCES}

[1] Y. Berkovich, Groups of prime power order, Vol. 1, Walter de Gruyter, Berlin-New York, 2008.

[2] Y. Berkovich and Z. Janko, Groups of prime power order, Vol. 3, Walter de Gruyter, Berlin-New York, 2011.

\section{Z. Janko}

Mathematical Institute

University of Heidelberg

69120 Heidelberg

Germany

E-mail: janko@mathi.uni-heidelberg.de

Received: 8.10.2015. 